\title{
Revisionen in der Kniegelenksendoprothetik
}

\author{
Steffen Oehme
}

\section{Zusammenfassung}

Knieendoprothetische Revisionsversorgungen sind heute häufige Operationen, die in vielen endoprothetisch tätigen Kliniken eine überproportionale Steigerungsrate aufweisen. Mit modernen Operationstechniken und der Anwendung von modernen modularen Prothesensystemen sind viele Wechselsituationen heute zu beherrschen und es können eben früh- wie spätfunktionelle Ergebnisresultate erreicht werden, die denen der primären Knieendoprothetik kaum nachstehen. Besonderes Augenmerk ist aber immer auf die logistischen Möglichkeiten in der Klinik und die vorgehaltenen Optionen des jeweiligen Prothesensystems zu richten, da gerade bei der Revisionschirurgie des Kniegelenks auch intraoperativ sich Situationen ergeben können, die eine Ausweitung des Eingriffs erforderlich werden lassen. Mit den Techniken des Trabecular Metal sind zusätzliche Optionen des Knochenaufbaus möglich, die auch in Kombination mit der Eigenund Fremdspongiosaplastik angewendet werden können.

\section{Zunahme der Knieendoprothesenrevision}

Die endoprothetische Versorgung des Kniegelenks ist inzwischen in vielen Kliniken ein Standardeingriff. Jährlich werden in Deutschland mehr als 150000 Kniegelenksendoprothesen implantiert. Die Zahl der Primäroperationen ist dabei kontinuierlich steigend und hat inzwischen fast die Anzahl der jährlich in

OP-JOURNAL 2010; 26: 28-31

(c) Georg Thieme Verlag KG Stuttgart · New York DOI http://dx.doi.org/10.1055/s-0030-1250209

\section{Revision Surgery in \\ Total Knee Arthroplasties}

Revision procedures for total knee arthroplasties are today frequent operations and exhibit an overproportional increase in many clinics specialising in joint replacements. With modern operating techniques and the use of modern modular prosthetic systems, many replacement situations can now be managed with both early and late functional results that are well comparable with those of primary knee arthroplasties. However, particular attention must always be paid to the logistic possibilities in the clinic and the available options of the respective prosthetic system since, especially in revision surgery of the knee, intraoperative situations can arise that necessitate an extension of the procedure. Additional options for bone replacement are possible with the techniques of trabecular metals, and these can also be employed in combination with autologous and allogenous spongiosaplasties.

Deutschland implantierten Hüftendoprothesen erreicht.

Dabei gilt in der Knieendoprothetik ebenso wie auch in der Hüftendoprothetik der Grundsatz, dass kein heute verfügbares Implantat eine unbegrenzte Standzeit haben wird. Mit den heute üblichen bikondylären Knieendoprothesensystemen, die inzwischen zum Standard der Knieendoprothetik herangereift sind, haben wir Standzeiten, die sich mit durchschnittlich 15 Jahren in einem guten Bereich bewegen.

Mit zunehmender Standzeit wird jedoch das Risiko für notwendige Revisionsope- rationen steigen und auch in der Knieendoprothetik sehen wir das Phänomen, dass bei jüngeren Patienten die Lebensdauer der Knieendoprothese eher etwas geringer sein wird als bei der Erstimplantation im höheren Lebensalter. Auch gilt in der Knieendoprothetik, dass das Revisionsrisiko für Frauen etwas geringer als für Männer anzusehen ist.

Wir werden also in den kommenden Jahren eine steigende Anzahl an Knieprothesenrevisionen in den Kliniken zu versorgen haben.

In unserem Hause konnten wir in den vergangenen Jahren bereits die Tatsache beobachten, dass bei kontinuierlichem Anstieg der primären Knieendoprothetik die Anzahl der jährlich zu versorgenden Revisionsoperationen überproportional ansteigend war.

Für diese Situation müssen wir uns in den Kliniken entsprechend rüsten und es stehen uns heute auch im Bereich der Knieendoprothetikrevision optimale Versorgungsmöglichkeiten für den $\mathrm{Pa}$ tienten zur Verfügung.

\section{Richtiger OP-Zeitpunkt}

Der Erfolg der Knieendoprothesenrevision hängt dabei ganz wesentlich von der Auswahl des richtigen Operationszeitpunkts ab. Dies gilt insbesondere, wenn es nach langjähriger Standzeit der primären Knieendoprothese $\mathrm{zu}$ den sog. Inlayschäden der Polyäthylengleitfläche gekommen ist (Abb.1). Durch regelmäßige radiologische Verlaufskontrollen lässt sich hier eine Schädigung des Polyäthyleninlays sicher diagnostizieren und bei rechtzeitiger Festlegung einer Revisionsoperation vor vollständigem Versagen der PE-Gleitfläche wird das Risiko von Schäden an den femoralen oder tibialen Prothesenkomponenten vermindert. Diese können beim alleinigen Wechsel der PE-Gleitfläche dann belassen werden. 


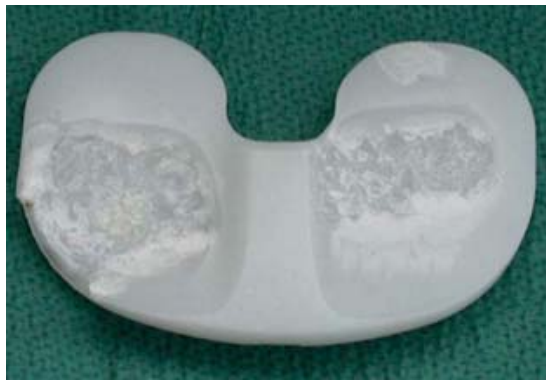

Abb. 1 PE Abrieb Schaden.

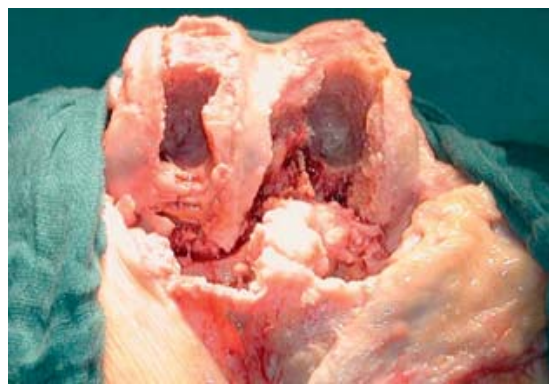

Abb. 4 Knochendefekte nach Prothesenentfernung.

Eine weitere Indikation für einen Wechsel einer Knieendoprothese besteht in den Fällen einer sich ausdehnenden Gonarthrose, die primär gegebenenfalls nur mit einer medialen Schlittenprothese versorgt worden war. Die medialen Schlittenprothesen haben inzwischen auch sehr exzellente Standzeiten, die an die Standzeit eines bikondylären Prothesensystems heranreichen. Limitiert werden die Standzeiten aber durch eine sich ausweitende Arthroseerkrankung in das laterale und/oder retropatelläre Kompartment des Kniegelenks. Bei dann wieder auftretenden Belastungsbeschwerden und einer erneuten Funktionseinschränkung des Kniegelenks besteht die Indikation für den Wechsel der Schlittenprothese auf ein bikondyläres System, gegebenenfalls auch mit Ersatz der Kniescheibenrückfläche.

Die Entfernung der Schlittenprothese kann bei Verwendung entsprechender Primärsysteme heute sehr schonend erfolgen und es ist kaum mit einem größeren Knochenverlust im Bereich des Femurs zu rechnen (Abb.2). Die tibialen Defekte nach Entfernung der medialen Tibiakomponente des Schlittens können ebenfalls mit einem bikondylären System heute gut wieder aufgebaut werden.

Regelmäßige radiologische Verlaufskontrollen sind aber auch nach primär bikondylär eingebrachten Knieendoprothesen-

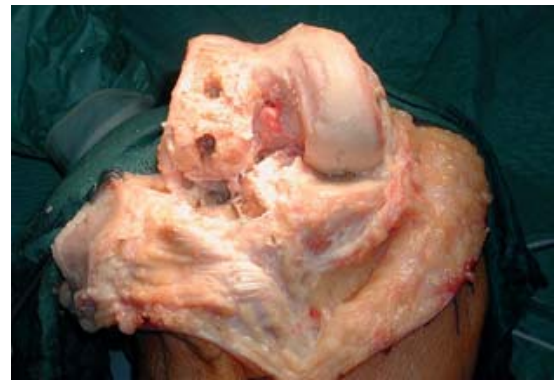

Abb. 2 Femur nach Entfernung einer medialen Schlittenkomponente.

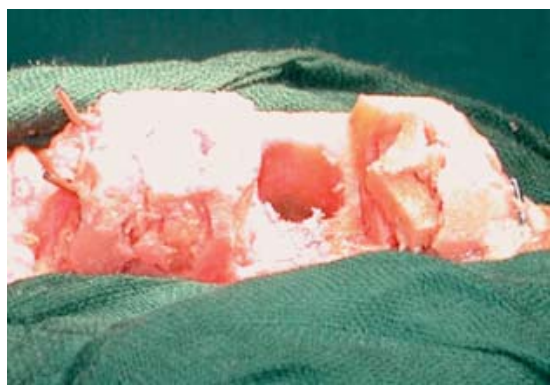

Abb. 5 Aufbau der Knochendefekte mit eigenem Knochen aus den Resektionsschnitten.

systemen unverzichtbar (Abb. 3). Dabei müssen zur exakten Beurteilung der aktuellen Röntgenaufnahmen immer auch die früheren Röntgenbilder vergleichsweise herangezogen werden können, denn nur ein direkter Vergleich der aktuellen Bilder mit vorhandenen früheren Aufnahmen kann hier einen ersten Anhalt für eine Lockerungsproblematik der Knieendoprothese ergeben.

Das Ziel all dieser Maßnahmen ist immer. einen optimalen Zeitpunkt für die operative Revision der Knieendoprothese festzulegen.

Bleibt eine Endoprothesenlockerung zu lange unerkannt, so besteht die große Gefahr der erheblichen knöchernen Defektbildung im Bereich der femoralen und/oder tibialen Prothesenlager.

\section{Aufbau von Knochendefekten}

Sind Knochendefekte erst einmal aufgetreten (Abb.4), so können diese bei den Revisionsoperationen zumindest partiell mit Knochen aus den Resektatschnitten des Kniegelenks aufgefüllt werden (Abb. 5).

In vielen Fällen muss aber auch auf Fremdknochen, der bspw. nach dem Marburger Knochenbanksystem in einer klinikeigenen Knochenbank vorgehalten werden kann, zurückgegriffen werden.

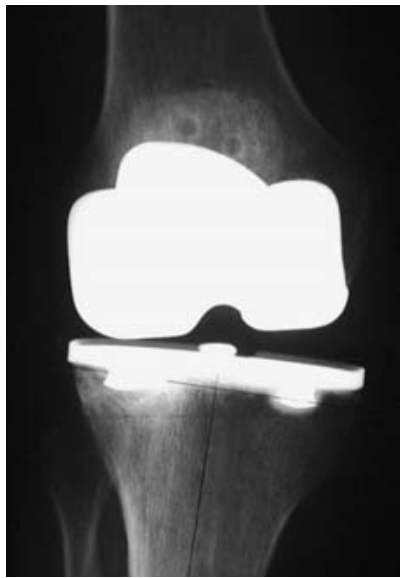

Abb. 3 Radiologischer Verlauf 14 Jahre nach Primär-OP.

Mehr noch als bei der primären Knieendoprothetik ist im Falle von Knieendoprothesenrevisionen eine exakte präoperative Planung auch anhand von Einbeinganzaufnahmen des Patienten erforderlich.

Im Vorfeld der Operation muss sich der Operateur über die bestehende Beinachse und gegebenenfalls erforderliche Korrekturmaßnahmen im Klaren sein. Die Rotationsausrichtung insbesondere der Femur-, aber auch der Tibiakomponente muss exakt festgestellt und für die Revision geplant werden, und für die spätere Funktion des Gelenks ist die exakte Wiederherstellung auch der Gelenklinie von großer Bedeutung.

\section{Auswahl geeigneter Implantate}

In Abhängigkeit vom Ausgangsbefund muss dann auch überlegt werden, ob die Implantation einer Revisionsknieendoprothese, die durch den eigenen Bandkapselapparat alleine geführt und gehalten werden kann, möglich ist oder aber ob auf sog. teilgekoppelte Knieendoprothesensysteme zurückgegriffen werden muss. Dies insbesondere in den Fällen, in denen größere Insuffizienzen im Bereich des Bandkapselapparats des Kniegelenks bereits bestehen.

Die teilgekoppelten Knieendoprothesen erfordern dabei regelhaft eine sog. Stielverlängerung in das Femur und in den Tibiaschaft hinein. Über diese Stielverlängerungen wird ein Teil der Krafteinleitung in knieferneren Regionen erreicht (Abb. 6).

Falls immer möglich, werden die Prothesenschäfte im Femur und in der Tibia zementfrei eingebracht, lediglich die Pro- 


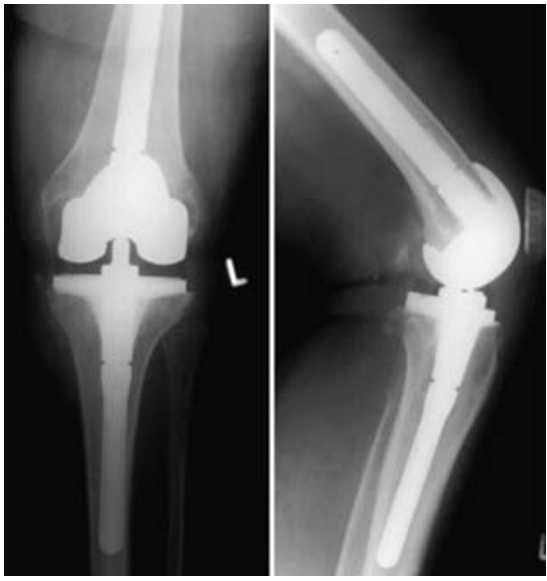

Abb. 6 Teilgekoppelte Knieendoprothese mit femoraler und tibialer Schaftverlängerung.

thesenkomponenten im Bereich der Femurkondylen und am Tibiaplateau selbst werden in zementierter Technik eingesetzt.

Letztendlich ist bei Wechselsituationen am Kniegelenk immer ein Prothesensystem in der Klinik vorzuhalten, das eine Anpassung der Knieendoprothese an die jeweils bestehende Ausgangssituation ermöglicht.

Die modernen modularen Knierevisionssysteme bieten diese Möglichkeit.

Ein weiterer Vorteil dieser Prothesensysteme ist der innerhalb eines solchen Systems unveränderte Aufbau der femoralen wie tibialen Prothesenanteile, sodass hier in schrittweiser Technik die Prothese auf eine zunehmende teilgekoppelte Variante aufgebaut werden kann. Alle modularen Prothesensysteme bieten darüber hinaus die Möglichkeit, knöcherne Defekte insbesondere im Bereich des Tibiaplateaus, aber auch distal an den Femurkondylen mit sog. Augmenten, d.h. Metallblöcken, die an die Prothese selbst fixiert werden, aufzubauen. Dies stellt in vielen Fällen eine gute Ergänzung zu den oben bereits beschriebenen Aufbautechniken mit Eigen- und Fremdknochen dar.

Gerade tibialseitig ist dabei eine exakte Ausrichtung der Prothesenkomponente auf dem Schienbeinkopf nur unter Verwendung von sog. Offset-Stems möglich. Diese können in $360^{\circ}$ freier Rotation distal an das Tibiaplateau angebracht werden und ermöglichen die optimale $\mathrm{Ab}$ deckung des Schienbeinkopfs durch die Prothesenkomponente.

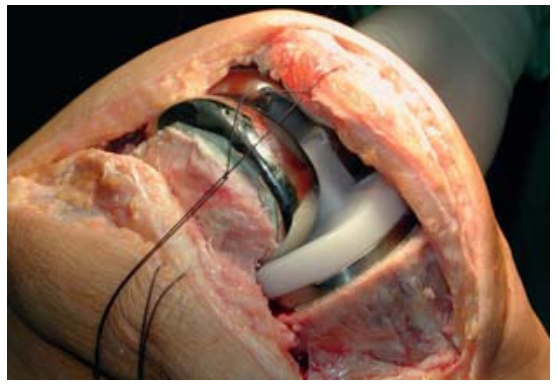

Abb. 7 Sichere Patellaführung.

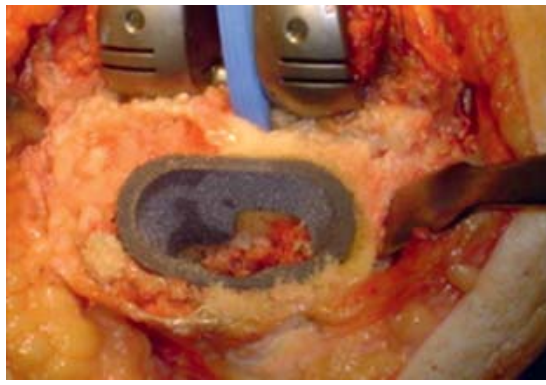

Abb. 8 Trabecular Metal Cone am Tibiakopf.

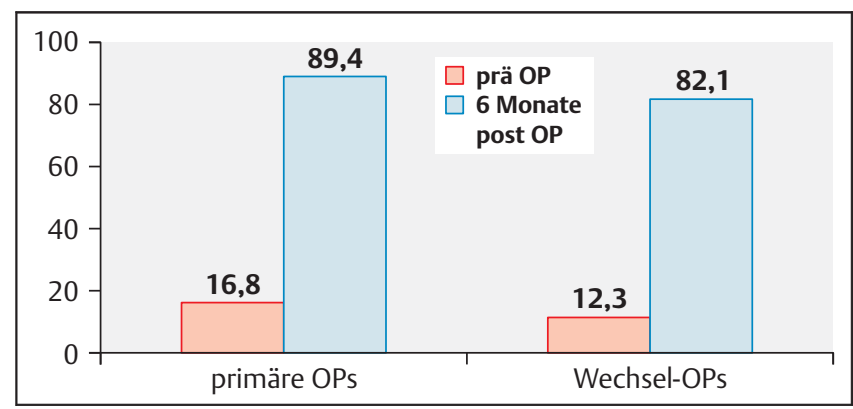

Abb. 9 Knee Society Score nach Knierevisionen.
Der Anwendungsbereich für solche Systeme geht bis hin zu der Versorgung von kniegelenksnahen Frakturen, die auch über ein teilgekoppeltes System stabilisiert und damit frühfunktionell belastbar versorgt werden können.

In Abhängigkeit von der Höhe des Polyäthyleninlays ist bei der Auswahl des Prothesendesigns auch auf eine sichere Führung der Kniescheibe und v.a. des Kniescheibenbands zu achten. Moderne Prothesensysteme zeigen hier im Bereich der PE-Gleitflächen entsprechende Aussparungen, sodass es nicht zu einem Impingement der Patellarsehne an den höheren Inlaykomponenten kommen kann (Abb. 7).

\section{Ergebnisse nach \\ Knieendoprothesenrevision}

In unserer Klinik haben wir die Ergebnisse nach knieendoprothetischen Versorgungen hinsichtlich der Funktion des Gelenks nach dem Knee Society Score bewertet und eine größere Anzahl solcher Versorgungen mit den funktionellen Ergebnissen nach primärer Operation verglichen.

Erreichen wir nach primärer knieendoprothetischer Versorgung mit 89,4 knee society score Punkten sehr gute Ergebnisse, so stehen die funktionellen Ergebnisse nach Wechseloperationen mit 82,1 Punkten nach dem Knee Society Score kaum unter den Ergebnissen der Primärversorgung (Abb. 9).

Weiterentwicklungen im Bereich der Materialtechnologie haben in den letzten Jahren neue Möglichkeiten des Defektaufbaus an den Knochenlagern erbracht.

Hier sei besonders auf das Trabecular Metal hingewiesen, ein Tantalmetall, welches sich durch eine gute Verformbarkeit und durch einen Elastizitätsmodul, der dem des menschlichen Knochens sehr ähnlich ist, auszeichnet.

Hat das Trabecular Metal zunächst in der Revisionschirurgie des Hüftgelenks Einzug gehalten und dort bereits seit nunmehr fast 10 Jahren sehr gute Ergebnisse gezeigt, so sind seit 2005 entsprechende Trabecular Metal-Blöcke auch in der Revisionschirurgie des Kniegelenks verfügbar (Abb. 8).

Diese sog. Trabecular Metal Cones gibt in verschiedener Formanpassung für den Defektaufbau am Schienbeinkopf und korrespondierend auch für einen Defektaufbau im Bereich der Femurkondylen. Dabei kann auch diese Technik des Defektaufbaus mit Eigen- und Fremdspongiosaplastiken kombiniert werden.

In die dann aufgebauten Defekte hinein wird die modulare Knieendoprothese eingesetzt, wie dies auch bei den anderen Revisionen ansonsten möglich wäre. 


\section{Gelenkrekonstruktion}

Bei Revisionen der Knieendoprothesen sei nochmals darauf hingewiesen, dass insbesondere eine exakte Wiedereinstellung der Flexions- und Extensionslücke für die spätere Funktion des Kniegelenks von großer Bedeutung ist.

Dabei muss man sich immer der Tatsache bewusst sein, dass eine gegebenenfalls erforderliche Nachresektion im Bereich der Tibia sowohl die Flexion- als auch die Extensionslücke des Kniegelenks beeinflusst, femoralseitig kann durch eine Veränderung der Prothesengröße und/oder eine Änderung der Resektionslinie die Flexion oder die Extension beeinflusst werden.

Es muss daher intraoperativ immer geprüft werden, ob die Flexions- und die Extensionslücke gut ausbalanciert ist und entsprechend des dann vorgefundenen Ergebnisses muss überlegt werden, ob gegebenenfalls eine distale Femuraugmentation mit Distalisierung der
Femurkomponente das gewünschte balancierte funktionelle Ergebnis ergeben kann, oder aber ob gegebenenfalls eine kleinere Femurkomponente und ein dickeres tibiales Polyäthyleninlay ein besseres funktionelles Resultat darstellt.

Insgesamt können auch nach knieendoprothetischen Revisionseingriffen heute sehr gute funktionelle Ergebnisse erreicht werden.

Die Patienten werden analog der Primärversorgung des Kniegelenks frühfunktionell nachbehandelt und haben in aller Regel bereits nach einer Woche ein gutes Gangbild mit 2 Unterarmstützen zumindest auf Stationsflurebene erreicht.

Für die Kliniken selbst, die Revisionsoperationen des Kniegelenks durchführen, besteht die Verpflichtung einer vorherigen Überprüfung der eigenen vorhandenen Logistik hinsichtlich der operativen Möglichkeiten und v.a. hinsichtlich der vor Ort vorhandenen Prothesenmaterialien. Da auch bei noch so exakter prä- operativer Planung intraoperativ immer Eventualitäten auftreten können, die jedenfalls eine Erweiterung des Eingriffs und damit auch die zusätzliche Verwendung von weiteren Endoprothesenmaterialien erforderlich werden lassen können. Mit den modernen modularen Knieendoprothesen-Revisionssystemen haben wir aber heute sog. Baukastensysteme zur Verfügung, die eine gute Anpassung der Knieendoprothese an die sich intraoperativ bietende Ausgangssituation ermöglichen.

\section{Literatur}

beim Verfasser

\section{Dr. med. Steffen Oehme}

Chefarzt

Orthopädie I

Ostseeklinik Damp GmbH

Seute-Deern-Ring 20

24351 Damp

steffen.oehme@damp.de 